\title{
Modeling and Mechanism of Rain-Wind Induced Vibration of Bundled Conductors
}

\author{
Chao Zhou and Yibing Liu \\ North China Electric Power University, School of Energy, Power and Mechanical Engineering, Beijing 102206, China \\ Correspondence should be addressed to Chao Zhou; zhouchao@ncepu.edu.cn
}

Received 28 April 2015; Accepted 23 August 2015

Academic Editor: Jussi Sopanen

Copyright ( 92016 C. Zhou and Y. Liu. This is an open access article distributed under the Creative Commons Attribution License, which permits unrestricted use, distribution, and reproduction in any medium, provided the original work is properly cited.

Under the certain rain-wind conditions, bundled conductors exhibit a rain-wind induced large-amplitude vibration. This type of vibration can cause the fatigue fractures of conductors and fatigue failures of spacers, which threaten the safety operation and serviceability of high-voltage transmission line. To reveal the mechanism of rain-wind induced vibration of bundled conductors, a series of 2-dimensional CFD models about the twin bundled conductors with rivulets are developed to obtain the curves of aerodynamic coefficients with the upper rivulet angle. The influences of the forward conductor's aerodynamic shielding and the upper rivulet's aerodynamic characteristics on the leeward conductor are discussed. Furthermore, a 2-dimensional 3DOF model for the rain-wind induced vibration of the leeward conductor is established. The model is solved by finite element method and Newmark method, and the effects of the wind velocity and the upper rivulet's motion on vibration amplitude of the leeward conductor are analyzed. By contrast with the wake-induced vibration, it can easily find that the characteristics of rain-wind vibration are obviously different from those of the wake-induced vibration, and the main reason of the rain-induced vibration may be due to the upper rivulet's motion.

\section{Introduction}

The planning ultra-high-voltage (UHV) transmission lines will cross the southwestern regions of China and one of the key technologies is how to solve vibration problems of conductors, especially on wet and windy days. Recent studies find that, besides icing galloping, large-amplitude oscillations may occur in the bundled conductors under the certain rainwind conditions, and the mechanism is still unknown. The effects of this phenomenon resulted in fatigue fractures of conductors, fatigue failures of spacers, and insulators, which threaten the safety operation and serviceability of the UHV transmission lines $[1,2]$.

Due to the high flexibility and low structural damping of the bundled conductors, the bundled conductors are sensitive to wind, icing, and rainfall. However, researchers were mainly concentrating on aeolian vibration, wake-induced vibration, and icing galloping and very little concerning about the oscillation problems of the UHV transmission lines in rainwind coupling field [3-5].

The first wind tunnel test on aerodynamic coefficients of the single conductor with different rainfall intensity was carried out by Kikuchi et al. [6]. The test result shows that the rainfall intensity has obvious effects on drag coefficient of the conductor in vertical direction. Li et al. $[7,8]$ proposed a simulation method for rain excitation acting on transmission tower-line system and established a model based on Kaimal spectrum and finite element method. The numerical studies show that the effects of rain excitation on the transmission tower-line system may not be neglected. Brahami et al. [2] discussed the effect of rainfall on corona-induced vibration of transmission lines and pointed out the vibration amplitude of the conductors subjected to rainfall was linearly increased with the precipitation intensity, up to roughly $25 \mathrm{~mm} / \mathrm{h}$, and then remained almost constant. Zhou et al. $[9,10]$ proposed a rain-wind induced vibration of single conductor with finite element method. By analytical results, he explained that the rain-wind vibration was a coupling action of vortex-induced vibration by the ionic wind and the external wind. The suspended droplets on the conductors amplified corona discharges with ionic wind, formed noncircular cross-sections.

Although various numerical models and experimental tests were studied to explain the phenomenon, the research works were limited to the rain-wind induced oscillation of 


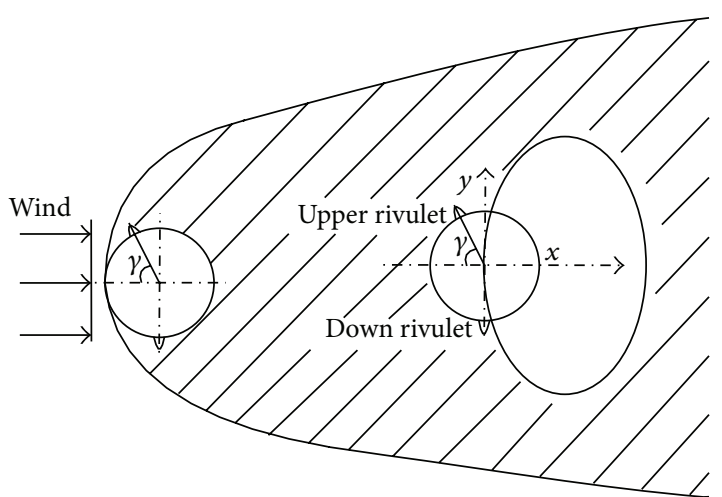

FIGURE 1: Oscillation of the leeward conductor under the rain-wind conditions.

a single conductor. To reveal the mechanism further, this research is to investigate aerodynamic characteristics of the bundled conductors with rivulet, validate a numerical model of the rain-wind vibration of the bundled conductor, and consider the variation of several factors such as rivulet's motion, wind velocity, drag, and lift coefficients.

\section{Rain-Wind Induced Vibration Model of Bundled Conductors}

Now consider oscillations of twin bundled conductors; evidently, the leeward conductor is submerged in the wake of the forward conductor. Under the certain rain-wind conditions, raindrops hitting the bundled conductors form an upper rivulet and a down rivulet, and the upper rivulet moved around the conductor surface with the oscillation of the conductor. As the motion of the forward conductor has little effects on vibration of the leeward conductor [11], the effects of the upper rivulet's motion coupled with aerodynamic shielding are only considered. Oscillation elliptical orbit of the leeward conductor is illustrated in Figure 1.

To simplify the analysis, the twin bundled conductors are reduced to a rigidly fixed forward cylinder and an elastically suspended leeward cylinder, and the effects of the down rivulet on the surface of the leeward cylinder are ignored. The state of the leeward conductor is described by a system of three equations of motion along $x, y$, and $\gamma$, and 3DOF vibration model of the leeward conductor is shown in Figure 2:

$$
\begin{aligned}
M \ddot{x}+c_{x} \dot{x}+k_{x} x & \\
= & \frac{1}{2} \rho U^{2} d\left(C_{D}(\phi+\gamma) \cos \phi-C_{L}(\phi+\gamma) \sin \phi\right), \\
M \ddot{y}+c_{y} \dot{y}+k_{y} y & \\
= & \frac{1}{2} \rho U^{2} d C_{D}(\phi+\gamma) \sin \phi \\
& +\frac{1}{2} \rho U^{2} d C_{L}(\phi+\gamma) \cos \phi, \\
m r \ddot{\gamma} & +m \cos \gamma(g-\ddot{y})+m \sin \gamma \ddot{x}=f_{\tau},
\end{aligned}
$$

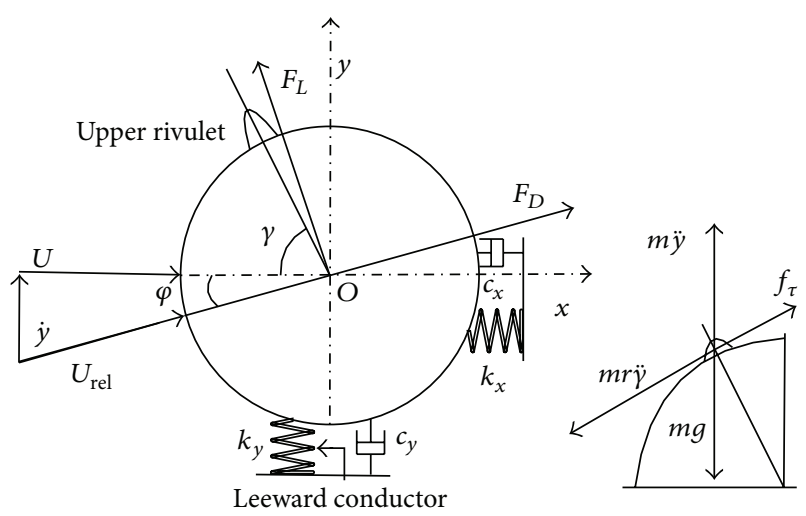

Figure 2: 3DOF vibration model of the leeward conductor.

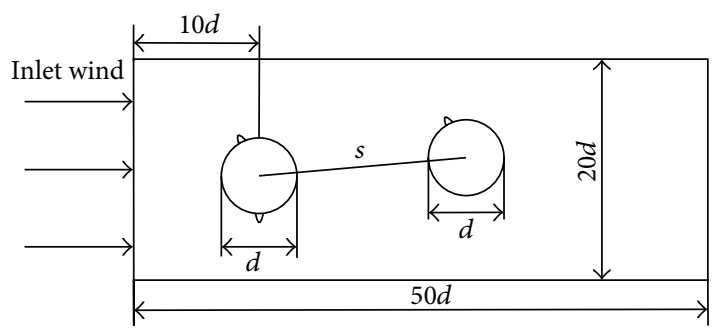

FIGURE 3: Computational domain of the bundled conductors with rivulets.

where $M, m$ are mass per unit length of the leeward conductor and the upper rivulet, respectively. $c_{x}, c_{y}$ are damping coefficients. $k_{x}, k_{y}$ are axial stiffness per unit length. $C_{D}, C_{L}$ are aerodynamic coefficient. $U, U_{\text {rel }}$ are local wind velocity and relative wind velocity. $\gamma$ is the upper rivulet angle, $\rho$ is density of the flowing medium (air with raindrops), $d$ is diameter of the leeward conductor, and $f_{\tau}$ is aerodynamic force of the upper rivulet.

\section{Aerodynamic Analysis of the Bundled Conductors with the Rivulets by CFD}

To simplify the analysis, we take the twin bundled conductors as example to study aerodynamic characteristics. Key parameters are as follows. The inlet wind velocity $U_{0}$ is $7 \sim 12 \mathrm{~m} / \mathrm{s}$, the Reynolds number of the flows is $\operatorname{Re}=(1.45 \sim 4.14) \times 10^{4}$, the air density $\rho$ is $1.225 \mathrm{~kg} / \mathrm{m}^{3}$, and the viscosity is $\nu=$ $1.45 \times 10^{-5} \mathrm{~m}^{2} / \mathrm{s}$. Figure 3 shows the computational domain of the bundled conductors with rivulets. The rectangular computational region is $50 \mathrm{~d} \times 20 \mathrm{~d}$, the upstream religion is $10 d$, the distance $s$ between two conductors is $10 d$, and the downstream region is $30 \mathrm{~d}$. The distance between the conductors and the upper/down boundary is $10 \mathrm{~d}$. The diameter $d$ of the conductor is $0.03 \mathrm{~m}$; the chord length $b$ of the rivulet is $0.008 \mathrm{~m}$ and the height $h$ is $0.006 \mathrm{~m}$. The boundary conditions are defined as follows: the left side is the velocity inlet and the flow velocity is uniform; the right side is the outflow and is a fully developed outlet boundary; the upper and down boundaries are symmetry; and the conducts 


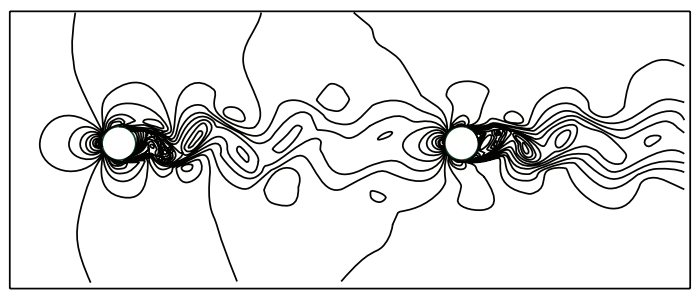

(a)

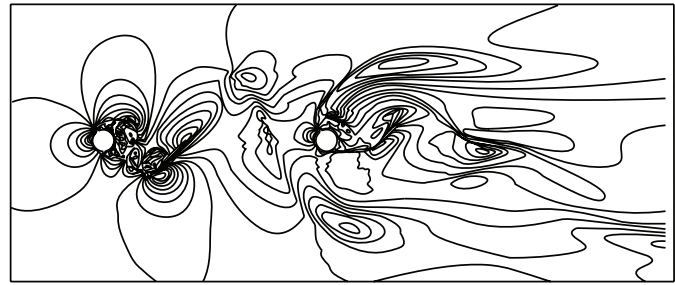

(b)

FIGURE 4: Comparison of the vorticity contours without rivulets (a) and with rivulets (b).

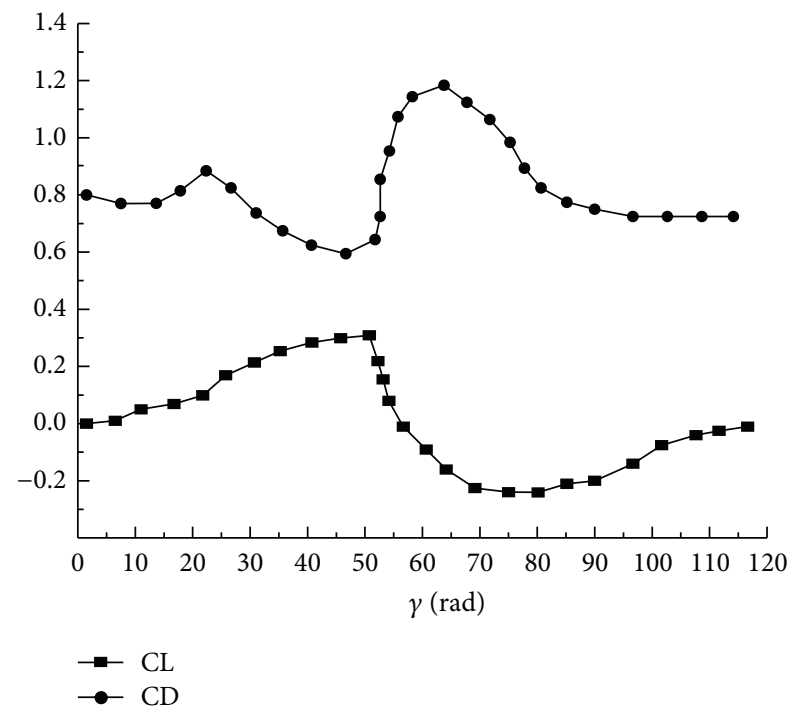

FIGURE 5: Aerodynamic coefficients versus the upper rivulet angle $\gamma$ of the leeward conductor.

surface is the wall. The SST $k-\omega$ turbulent model based on the RANS method is used to the conductor velocity.

The vorticity contours of the twin bundled conductors without the rivulets are shown in Figure 4(a), and the rivulets are shown in Figure 4(b). By comparing these curves of the vorticity contours, we find that the uniformity of the axial vorticity component in Figure 4(b) seems to be easier to break down, and the leeward conductor aerodynamic shielding in Figure 4(b) is more complex than in Figure 4(a). The recirculation zone behind the rivulets is the smaller one where the flow separates from the rivulets and then reattaches to the conductor surfaces at the back, and accordingly $C_{D}$ and St undergo sudden changes at a certain angle (see Figure 5). Similar observation is obtained in the experiment on a circular cylinder with two tripping rods by Mahbub Alam et al. [12].

The drag and lift coefficients of the leeward conductor with the upper rivulet are calculated by CFD method, and the coefficients can be expressed as function of the angle $\gamma$ as shown in Figure 5. In comparison with experimental test [13], the aerodynamic characteristics of the leeward conductor with the upper rivulet are obviously different with aerodynamic shielding of the leeward conductor by the forward conductors. It is necessary to point out that

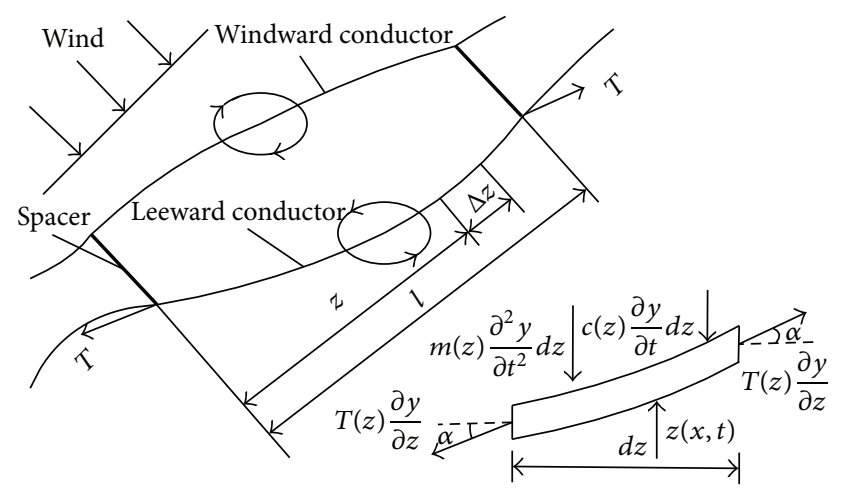

FIGURE 6: Mechanical model of the twin bundled conductors.

the drag and lift coefficients may be overestimated, and the lack of the third dimension makes vortex strength more easily be exaggerated. Therefore, the obtained aerodynamic coefficients of the leeward conductor need to be limited to a range of wind velocity $0 \sim 15 \mathrm{~m} / \mathrm{s}$, and the impact of the upper rivulet is likely to be greater than it actually is.

\section{Numerical Solution Method for Rain-Wind Induced Vibration of the Bundled Conductor}

The twin bundled conductors are flexure stocks-hinge structure, and bending stiffness varies within $16 \mathrm{~N} / \mathrm{m}^{2} \sim 577 \mathrm{~N} / \mathrm{m}^{2}$ [14]. In order to facilitate the analysis, the twin bundled conductors are simplified as slender solid cylinders with unified geometry and material properties along their longitudinal direction, and the bending stiffness is ignored. Figure 6 shows mechanical model of the twin bundled conductors.

Combining (1), (2), and (3), the leeward conductor is divided into discrete units by finite element method. The rainwind vibration equations can be rewritten as follows:

$$
\begin{gathered}
M(z) \frac{\partial^{2} x(z, t)}{\partial t^{2}}+c_{x}(z, t) \frac{\partial x(z, t)}{\partial t} \\
-\frac{\partial}{\partial t}\left(T(z) \frac{\partial x(z, t)}{\partial z}\right)=f_{x}, \\
M(z) \frac{\partial^{2} y(z, t)}{\partial t^{2}}+c_{y}(z, t) \frac{\partial y(z, t)}{\partial t}
\end{gathered}
$$




$$
\begin{gathered}
-\frac{\partial}{\partial t}\left(T(z) \frac{\partial y(z, t)}{\partial z}\right)=f_{y}, \\
m r \ddot{\gamma}+m \cos \gamma\left(g-\frac{\partial^{2} y(z, t)}{\partial t^{2}}\right) \\
+m \sin \gamma \frac{\partial^{2} x(z, t)}{\partial t^{2}}=f_{\tau},
\end{gathered}
$$

where $0 \leq z \leq l, t>0, M(z)$ is mass per unit length, $x(z, t)$, $y(z, t)$ are the displacements, $c_{x}(z), c_{y}(z)$ are the damping coefficients, $T(z)$ is tension force, and

$$
\begin{aligned}
& f_{x}=\frac{1}{2} \rho U^{2} d\left(C_{D}(\phi+\gamma) \cos \phi-C_{L}(\phi+\gamma) \sin \phi\right), \\
& f_{y}=\frac{1}{2} \rho U^{2} d\left(C_{D}(\phi+\gamma) \sin \phi+C_{L}(\phi+\gamma) \cos \phi\right) .
\end{aligned}
$$

The boundary displacements conditions are as follows:

$$
\mathrm{BC}(u): \begin{cases}z=0, & \left.z(0, t)\right|_{t>0}=0 \\ z=l, & \left.z(l, t)\right|_{t>0}=0 .\end{cases}
$$

The boundary forces conditions are as follows:

$$
\operatorname{BC}(p): \begin{cases}z=0, & \left.\sigma(0, t)\right|_{t>0}=\frac{T}{A} \\ z=l, & \left.\sigma(l, t)\right|_{t>0}=\frac{T}{A} .\end{cases}
$$

Take an approximate solution of the problem as

$$
\begin{aligned}
& x^{e}(z, t)=\sum_{i=1}^{n} \phi_{i}(z) v_{i}(t), \\
& y^{e}(z, t)=\sum_{i=1}^{n} \vartheta_{j}(z) \chi_{i}(t),
\end{aligned}
$$

where $\phi_{i}(z), \vartheta_{j}(z)$ are polynomial interpolation function, $v_{i}(t), \chi_{i}(t)$ represent the values of function (8) at the nodes, and $n$ is the number of liberty degrees.

The typical element pondered residual equation can be written as follows:

$$
\begin{aligned}
& \int^{e} R(z, t ; a) \phi_{i}(z) d z=0, \\
& \int^{e} R(z, t ; a) \vartheta_{j}(z) d z=0,
\end{aligned}
$$

where

$$
R(z, t ; a)=\left\{\begin{array}{l}
M(z) \frac{\partial^{2} x(z, t)}{\partial t^{2}}+c_{x}(z, t) \frac{\partial x(z, t)}{\partial t}-\frac{\partial}{\partial t}\left(T(z) \frac{\partial x(z, t)}{\partial z}\right)-f_{x} \\
M(z) \frac{\partial^{2} y(z, t)}{\partial t^{2}}+c_{y}(z, t) \frac{\partial y(z, t)}{\partial t}-\frac{\partial}{\partial t}\left(T(z) \frac{\partial y(z, t)}{\partial z}\right)-f_{y}
\end{array}\right.
$$

and (4) is rewritten as follows:

$$
\begin{aligned}
& \int_{i}^{e} \phi_{i}^{e}(z) M(z) \frac{\partial^{2} x(t)}{\partial t^{2}} d z+\int^{e} \phi_{i}^{e}(z) c_{x}(z) \frac{\partial x(t)}{\partial t} d z \\
& +\int^{e} \frac{d \phi_{i}^{e}(z)}{d z} T(z) \frac{\partial x(t)}{\partial z} d z=\int^{e} f_{x}(z, t) \phi_{i}^{e}(z) d z \\
& +\left.T(z) \frac{\partial x(t)}{\partial z} \phi_{i}^{e}(z)\right|_{z_{1}} ^{z_{n}}, \\
& \int_{j}^{e} \vartheta_{j}^{e}(z) M(z) \frac{\partial^{2} y(t)}{\partial t^{2}} d z+\int_{j}^{e} \vartheta_{j}^{e}(z) c_{y}(z) \frac{\partial y(t)}{\partial t} d z \\
& +\int^{e} \frac{d \vartheta_{j}^{e}(z)}{d z} T(z) \frac{\partial y(t)}{\partial z} d z=\int^{e} f_{y}(z, t) \vartheta_{i}^{e}(z) d z \\
& +\left.T(z) \frac{\partial y(t)}{\partial z} \vartheta_{j}^{e}(z)\right|_{z_{1}} ^{z_{n}} .
\end{aligned}
$$

Substituting (11) into (4) leads to

$$
\begin{aligned}
& {\left[M_{x}\right]^{e} \frac{d^{2} v(t)}{d t^{2}}+\left[C_{x}\right]^{e} \frac{d v(t)}{d t}+\left[K_{x}\right]^{e} v(t)} \\
& =F_{x}^{e}(z, t), \\
& {\left[M_{y}\right]^{e} \frac{d^{2} \vartheta(t)}{d t^{2}}+\left[C_{y}\right]^{e} \frac{d \vartheta(t)}{d t}+\left[K_{y}\right]^{e} \vartheta(t)} \\
& =F_{y}{ }^{e}(z, t),
\end{aligned}
$$

where

$$
\begin{aligned}
& M_{x}^{e}=\int \phi_{i}^{e}(z) m(z) \phi_{j}^{e}(z) d z, \\
& M_{y}^{e}=\int^{e} \vartheta_{i}^{e}(z) m(z) \vartheta_{j}^{e}(z) d z, \\
& C_{x}^{e}=\int^{e} \phi_{i}^{e}(z) C(z) \phi_{j}^{e}(z) d z, \\
& C_{y}^{e}=\int^{e} \vartheta_{i}^{e}(z) C_{y}(z) \vartheta_{j}^{e}(z) d z,
\end{aligned}
$$




$$
\begin{aligned}
& K^{e}{ }_{x}=\int \frac{d \phi_{i}^{e}(z)}{d z} T(z) \frac{d \phi_{j}^{e}(z)}{d z} d z, \\
& K_{y}^{e}=\int^{e} \frac{d \vartheta_{i}^{e}(z)}{d z} T(z) \frac{d \vartheta_{j}^{e}(z)}{d z} d z .
\end{aligned}
$$

After assembling all elements

$$
\begin{aligned}
& M_{x} \ddot{v}_{t}+C_{x} \dot{v}_{t}+K_{x} v_{t}=F_{x}(t), \\
& M_{y} \ddot{\chi}_{t}+C_{y} \dot{\chi}_{t}+K_{y} \dot{\chi}_{t}=F_{y}(t), \\
& m r \ddot{\gamma}+m \cos \gamma\left(g-\vartheta(z) \ddot{\chi}_{t}\right)+m \sin \gamma \phi(z) \ddot{v}_{t}=f_{\tau} .
\end{aligned}
$$

The system of (14) can be numerically solved by Newmark method as follows:

$$
\begin{aligned}
& \dot{\sigma}_{t+\Delta t}=\dot{\sigma}_{t}+\left[(1-\beta) \ddot{\sigma}_{t}+\beta \ddot{\sigma}_{t+\Delta t}\right] \Delta t, \\
& \sigma_{t+\Delta t}=\sigma_{t}+\dot{\sigma}_{t} \Delta t+\left[\left(\frac{1}{2}-\alpha\right) \ddot{\sigma}_{t}+\alpha \ddot{\sigma}_{t+\Delta t}\right] \Delta t^{2},
\end{aligned}
$$

$\sigma=v, \chi, \gamma$, and $\alpha, \beta$ determined with accuracy and stability of integration. By solving the system of (14), it is possible to find dynamic response and vibration amplitude and reveal mechanism of rain-wind vibration of the bundled conductors.

\section{Case Studies}

In order to reveal the mechanism of rain-wind induced vibration of bundled conductors, a $220 \mathrm{KV}$ twin bundled conductor was taken as an example. The main parameters of the twin bundled conductor are as follows: the span between two towers is $500 \mathrm{~m}$ and the subspan between two spacers is $70 \mathrm{~m}$, and the diameter of the conductor $d$ is $0.03 \mathrm{~m}$, and mass per unit length $M$ is $1.36 \mathrm{~kg} / \mathrm{m}$, and tension force $T$ at each spacer is $20 \mathrm{kN}$, and the spacing $s$ between the twin bundles is $10 \mathrm{~d}$. We obtain the aerodynamic coefficients of the leeward conductor from the curves of Figure 5 and applied them into the above proposed numerical method to study the effects of wind velocity and angle of the upper rivulet on rain-wind induced vibration of the leeward conductor.

\subsection{Effects of Wind Velocity on Rain-Wind Induced Vibration} of the Leeward Conductor. According to the large amount of field data, the rain-wind vibrations of the twin bundled conductor often occur at the wind velocity range of $4 \mathrm{~m} / \mathrm{s} \sim$ $16 \mathrm{~m} / \mathrm{s}$ and the rainfall rate from light to moderate [15]. To simplify analysis, in this section, we investigate the effect of different velocities ( $v=6 \mathrm{~m} / \mathrm{s}, v=10 \mathrm{~m} / \mathrm{s}$, and $v=12 \mathrm{~m} / \mathrm{s}$ ) on rain-wind induced vibration of the leeward conductor. As shown in Figure 7, the $X$-direction displacements of the leeward conductor at the point of middle subspan vary differently with different wind velocities. The displacement of the leeward conductor increases with the increasing of velocity, and the peak displacement is almost 13 times as big as the diameter $d$ of the leeward conductor, for the wind velocity of $12 \mathrm{~m} / \mathrm{s}$.

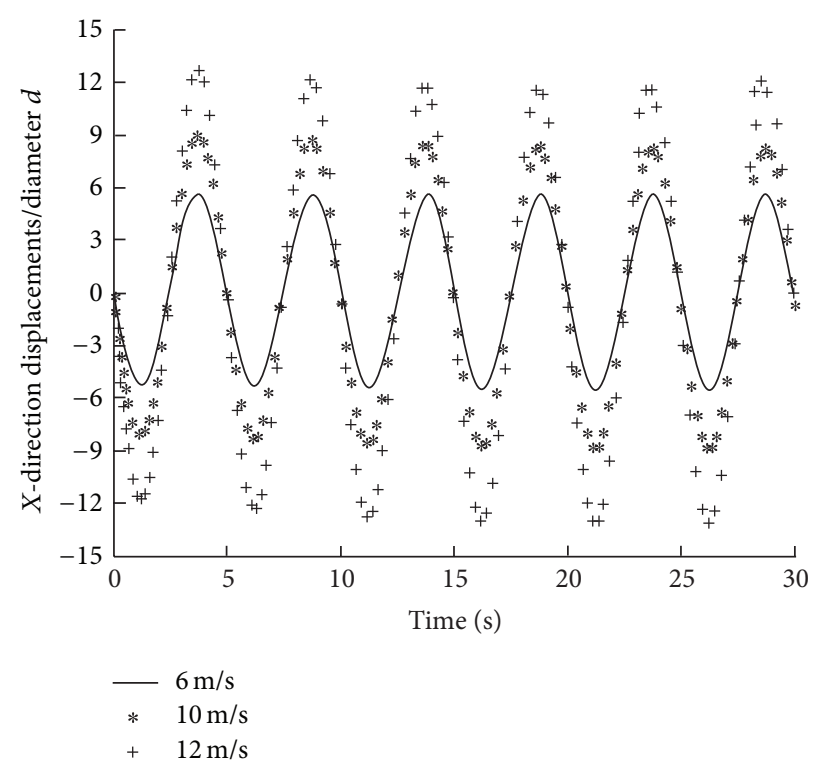

FIgURE 7: $X$-components displacements of the leeward conductor at the point of middle subspan.

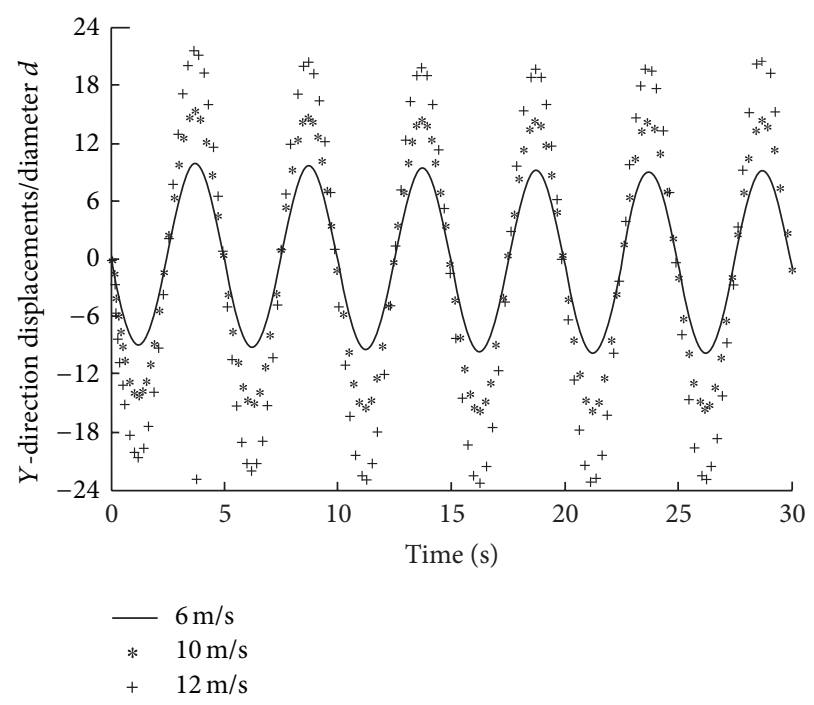

FIGURE 8: $Y$-components displacements of the leeward conductor at the point of middle subspan.

The $X$-direction displacements of the leeward conductor at the point of middle subspan vary differently with different wind velocities (Figure 8). The displacement of the leeward conductor increases with the increasing of velocity, and the peak displacement is almost 23 times as big as the diameter $d$ of the leeward conductor, for the wind velocity of $12 \mathrm{~m} / \mathrm{s}$. And Figure 9 shows the leeward conductor oscillation with the elliptical orbits.

These results mainly are in agreement with numerical analysis results of wake-induced vibration carried out by $\mathrm{Li}$ [16], while the $Y$-direction displacements are increased and the elliptical orbits have different orients. The difference can be explained by the facts that the forward conductor has 


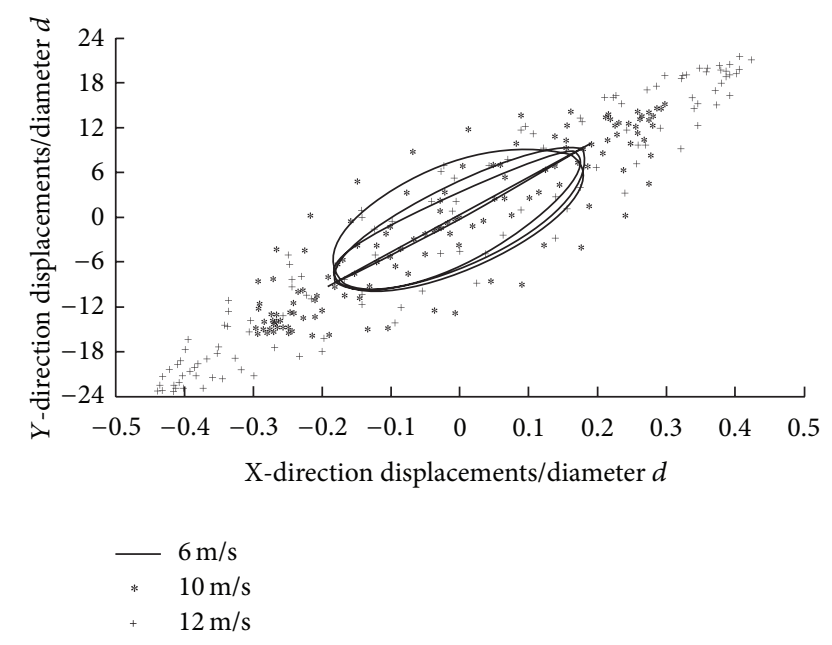

FIGURE 9: Leeward conductor displacements with the elliptical orbits.

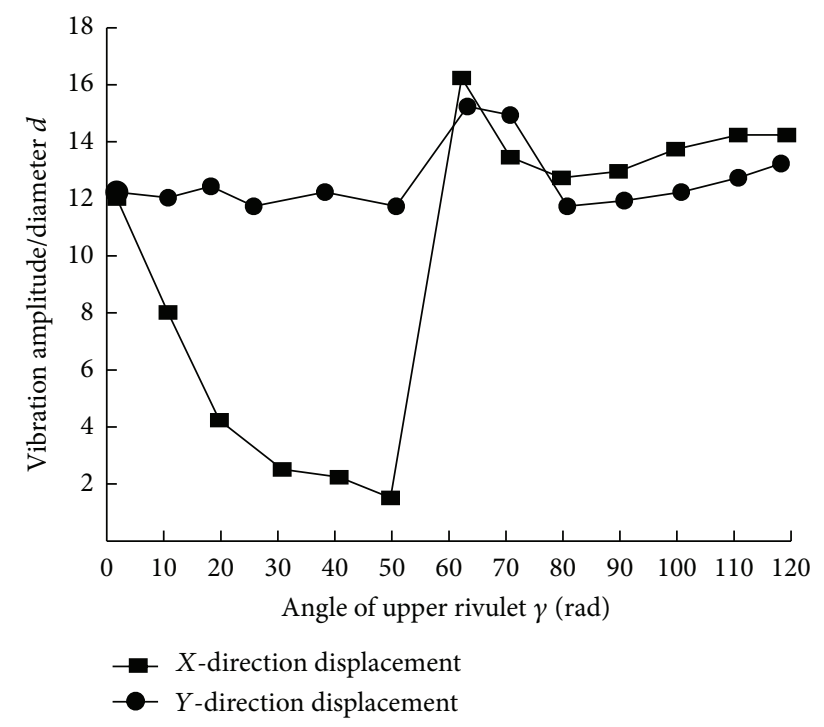

FIGURE 10: Effects of the angle $\gamma$ of the upper rivulet on vibration amplitude of the leeward conductor.

little effect on the flow field of around the leeward conductor and the upper rivulet has obvious effect on aerodynamic characteristics of the leeward conductor.

5.2. Effects of Angle of the Upper Rivulet on Vibration Amplitude of the Leeward Conductor. Figure 10 shows the vibration amplitude of the leeward conductor at $X$-direction and $Y$ direction versus angle $\gamma$ of the upper rivulet, for the wind velocity of $6 \mathrm{~m} / \mathrm{s}$ and damping coefficients $c_{x}=c_{y}=0.1$. From the comparison of these two curves, it can be concluded that when $\gamma<50^{\circ}$, the upper rivulet has little effects on flow field of the leeward conductor. A few smaller vortexes generated behind the upper rivulets, and the vortexes promoted the flow field of the leeward conductor transmitting from laminar flow to turbulent flow. The $X$-direction displacement of the leeward conductor decreases with the increasing of angle $\gamma$, the upper rivulet, while $Y$-direction displacement has no obvious changes. For $50^{\circ}<\gamma<80^{\circ}$, due to the fact that a critical zone occurs at the curves of aerodynamic coefficients, the upper rivulet has a substantial oscillation and its position is variable with a large range. Vibration amplitudes of the leeward conductor have obvious increases in both $X$-direction (peak displacement nearly 16 times of diameter $d$ ) and $Y$ direction (peak displacement nearly 15 times of diameter $d$ ). When $\gamma>80^{\circ}$, the upper rivulet only affects the nearby flow field of the leeward conductor and vibration amplitudes of the leeward conductor are gradually stabilized.

\section{Conclusions}

In order to investigate the mechanism of rain-wind induced vibration of bundled conductors, a series of researches are carried out. Firstly, 2-dimensional CFD models of twin bundled conductors with rivulets are computed to obtain the curves of aerodynamic coefficients versus the positions of upper rivulets. Secondly, the influences on leeward conductor aerodynamic shielding caused by forward conductor and aerodynamic characteristics caused by the upper rivulet are analyzed. Thirdly, a 2-dimensional 3DOF model of rainwind induced vibration of the leeward conductor is created. By contrast with the wake-induced vibration of leeward conductors, the effects of the wind velocity and the upper rivulet's motion on vibration amplitude are studied based on finite element method and Newmark method. The results indicate that the upper rivulet' motion is the main reason of the vibration, and characteristics of the vibrations obviously differ with the wake-induced vibrations.

It should be noted that the proposed analytical method based on 2D aerodynamic CFD results is still a preliminary method. The vortex strength tends to be exaggerated when third dimension is neglected. Some assumptions used in this research may be released in the further study.

\section{Conflict of Interests}

The authors declare that there is no conflict of interests regarding the publication of this paper.

\section{Acknowledgments}

This project is supported by the National Natural Science Foundation of China (Grant no. 51575180 and no. 51205128), the Beijing Natural Science Fund (Grant no. 8152027), and the Fundamental Research Funds for the Central Universities (Grant no. 2014ZD07).

\section{References}

[1] Y.-B. Shu and W.-L. Zhang, "Research of key technologies for UHV transmission," Proceedings of the Chinese Society of Electrical Engineering, vol. 27, no. 31, pp. 1-6, 2007.

[2] M. Brahami, A. Gourbi, A. Tilmatine, and L. Dascalescu, "Numerical analysis of the induced corona vibrations on highvoltage transmission lines affected by rainfall," IEEE Transactions on Power Delivery, vol. 26, no. 2, pp. 617-624, 2011. 
[3] S. H. Wang, X. L. Jiang, and C. X. Sun, "Study status of conductor galloping on transmission line," High Voltage Engineering, vol. 31, no. 10, pp. 11-14, 2005.

[4] H. N. Li and H. F. Bai, "State-of-the-art review on studies of disaster resistance of high-voltage transmission tower-line systems," China Civil Engineering Journal, vol. 40, no. 2, pp. 39-45, 2007.

[5] D. Sumner, "Two circular cylinders in cross-flow: a review," Journal of Fluids and Structures, vol. 26, no. 6, pp. 849-899, 2010.

[6] N. Kikuchi, Y. Matsuzaki, T. Yukino, and H. Ishida, "Aerodynamic drag of new-design electric power wire in a heavy rainfall and wind," Journal of Wind Engineering and Industrial Aerodynamics, vol. 91, no. 1-2, pp. 41-51, 2003.

[7] H.-N. Li, Y.-M. Ren, and H.-F. Bai, "Rain-wind-induced dynamic model for transmission tower system," Proceedings of the Chinese Society of Electrical Engineering, vol. 27, no. 30, pp. 4348, 2007.

[8] H. F. Bai and H. N. Li, "Dynamic response of overhead transmission lines to oscillation caused by wind or rainfalls loads," Power System Technology, vol. 33, no. 2, pp. 36-40, 2009.

[9] C. Zhou, Y. B. Liu, and X. M. Rui, "Mechanism and characteristic of rain-induced vibration on high-voltage transmission line," Journal of Mechanical Science and Technology, vol. 26, no. 8, pp. 2505-2510, 2012.

[10] C. Zhou and Y.-P. Liu, "Numerical analysis of rain-wind induced vibration on conductor by finite element method," Applied Mechanics and Materials, vol. 105-107, pp. 151-154, 2012.

[11] C. B. Rawlins, A. R. Hard, and R. Lkegami, Transmission Line Reference Book: Wind Induced Conductor Motion, Electric Power Research Institute, Palo Alto, Calif, USA, 1979.

[12] M. Mahbub Alam, Y. Zhou, J. M. Zhao, O. Flamand, and O. Boujard, "Classification of the tripped cylinder wake and bistable phenomenon," International Journal of Heat and Fluid Flow, vol. 31, no. 4, pp. 545-560, 2010.

[13] R. L. Wardlaw and K. R. Cooper, "Mechanism and alleviation of wind-induced structural vibration," in Proceedings of the 2 nd Symposium on Applications of Solid Mechanics, pp. 369-399, McMaster University, Hamilton, Canada, June 1974.

[14] J. Vecchiarelli, I. G. Currie, and D. G. Havard, "Computational analysis of aeolian conductor vibration with a stockbridge-type damper," Journal of Fluids and Structures, vol. 14, no. 4, pp. 489509, 2000.

[15] S.-R. Tang, W.-L. Chen, and H. Li, "Investigation on rainwind-induced vibration of stay cables based on numerical simulations," Engineering Mechanics, vol. 29, no. 3, pp. 124-132, 2012.

[16] Y. S. Li, Study on Aeolian vibration and subspan oscillation of bundle conductor [Ph.D. thesis], Huazhong University of Science and Technology, Wuhan, China, 2011. 


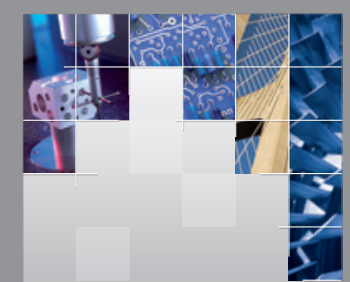

\section{Enfincering}
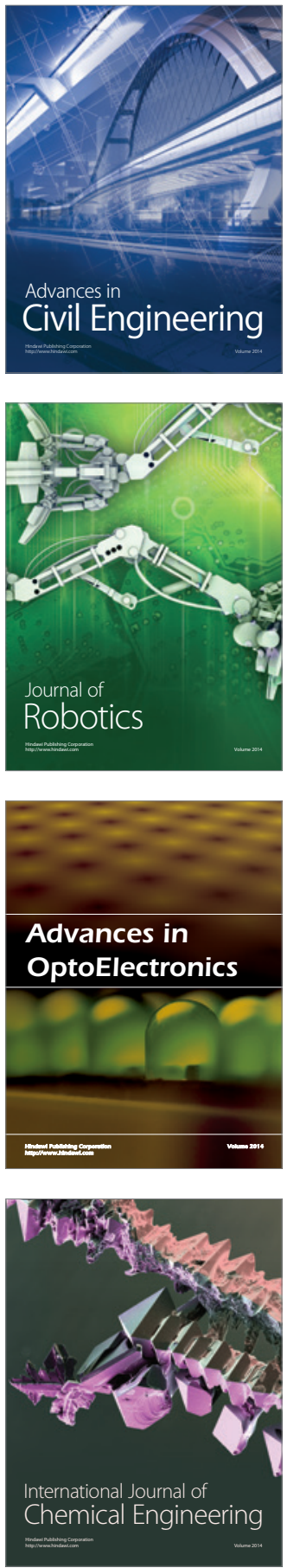

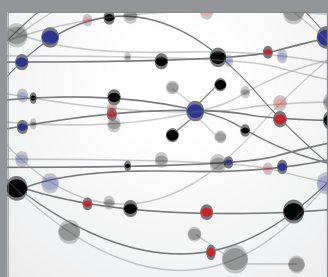

The Scientific World Journal

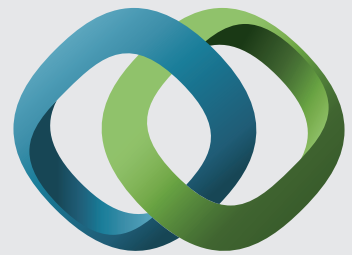

\section{Hindawi}

Submit your manuscripts at

http://www.hindawi.com
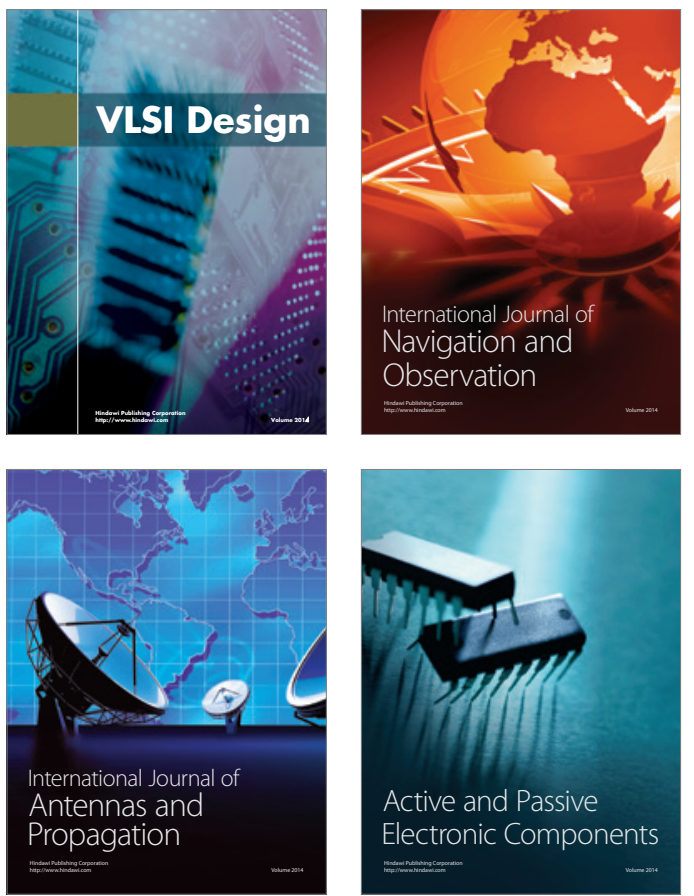
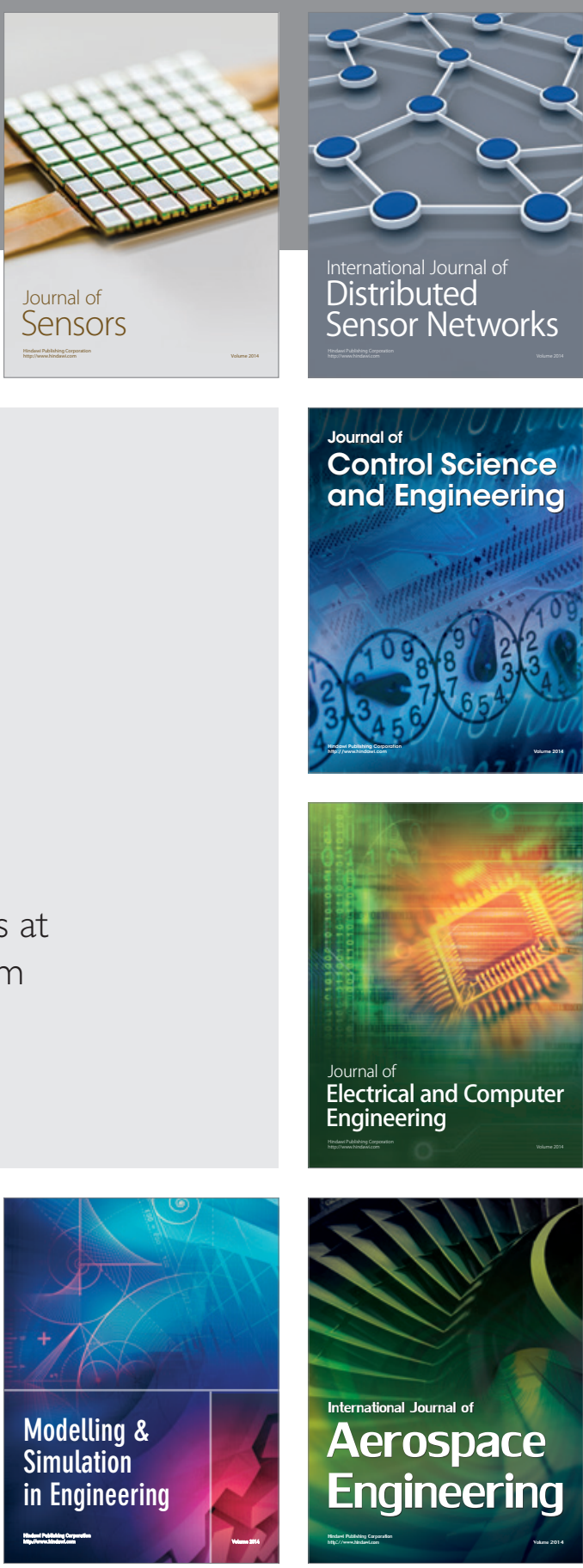

International Journal of

Distributed

Sensor Networks

Journal of

Control Science

and Engineering
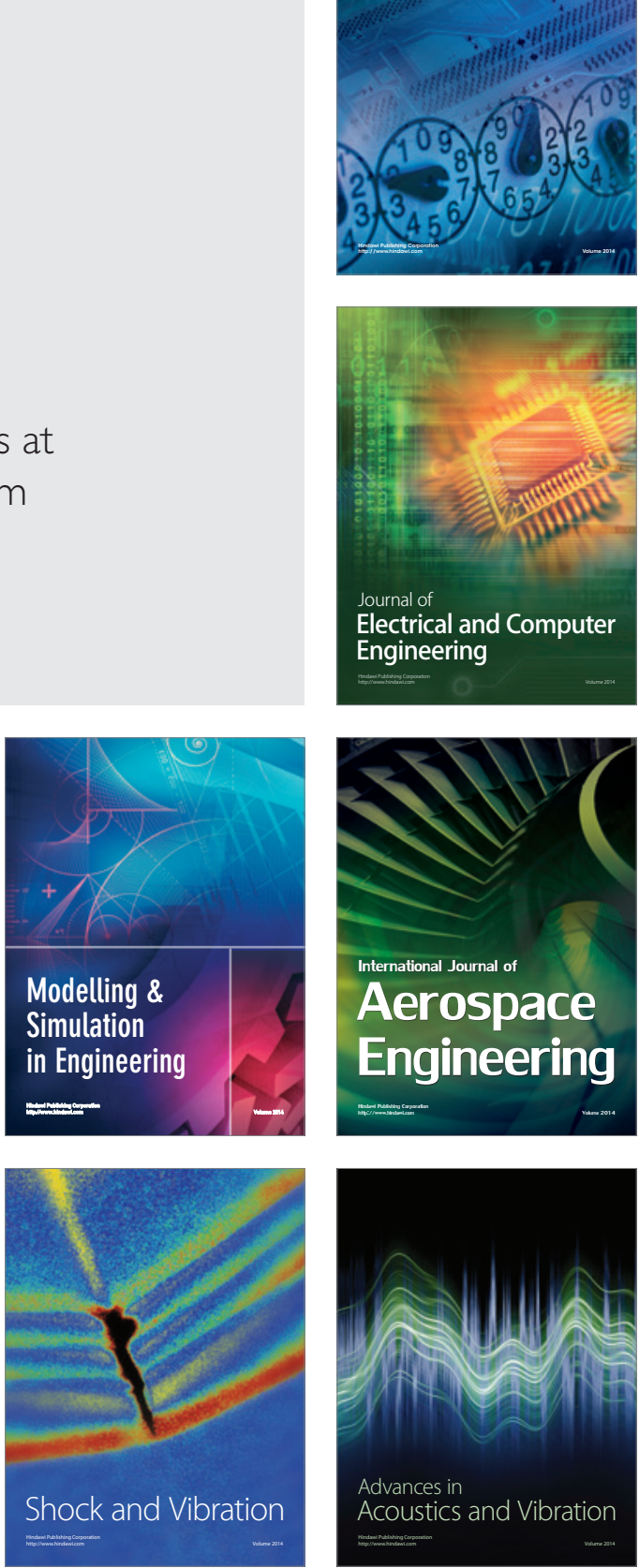